\title{
Minimal Surfaces in Euclidean 3-Space and Their Mean Curvature 1 Cousins in Hyperbolic 3-Space
}

\author{
SHOICHI FUJIMORI \\ Department of Mathematics, Kobe University, Kobe 657-8501, Japan \\ Manuscript received on February 4, 2003; accepted for publication on June 4, 2003; \\ presented by MANFREDO DO CARMO
}

\begin{abstract}
We show that the Hopf differentials of a pair of isometric cousin surfaces, a minimal surface in euclidean 3-space and a constant mean curvature (CMC) one surface in the 3-dimensional hyperbolic space, with properly embedded annular ends, extend holomorphically to each end. Using this result, we derive conditions for when the pair must be a plane and a horosphere.
\end{abstract}

Key words: minimal surfaces, CMC 1 cousins, hyperbolic space.

\section{INTRODUCTION}

As there is a way to deform simply-connected CMC 1 surfaces in hyperbolic 3-space $\mathbb{H}^{3}$ to minimal surfaces in Euclidean 3-space $\mathbb{R}^{3}$ (Umehara and Yamada 1992), one might expect that there exist cousins in these two spaces that are not simply-connected. However, although there are now many known examples of minimal surfaces in $\mathbb{R}^{3}$ and also CMC 1 surfaces in $\mathbb{H}^{3}$ (see, for example, Bryant 1987, Rossman et al. 1997, 2001, Sá Earp and Toubiana 2001, Yu 2001, Umehara and Yamada 1993), and although non-simply-connected cousins pairs are easily found, such a pair of surfaces with embedded ends is yet to be found. Our purpose is to investigate whether such a pair can exist. Toward this goal, we apply recent results in Collin et al. 2001 about the behavior of embedded CMC 1 ends in $\mathbb{H}^{3}$ to give various conditions under which such a pair cannot exist.

\section{RESULTS}

Let $D \subset \mathbb{C}$ be a simply-connected domain in the complex plane. Fix a point $z_{0} \in D$. Let $g$ be a meromorphic function on $D$ and $\omega$ a holomorphic 1-form on $D$ such that $\omega$ has a zero of order $2 k$

E-mail: fujimori@math.kobe-u.ac.jp 
if and only if $g$ has a pole of order $k$ and so that $\omega$ has no other zeros. Set

$$
\Phi_{0}(z):=\operatorname{Re} \int_{z_{0}}^{z}\left(1-g^{2}, i\left(1+g^{2}\right), 2 g\right) \omega .
$$

Then $\Phi_{0}: D \rightarrow \mathbb{R}^{3}$ is a minimal immersion with induced metric

$$
\Phi_{0}^{*}\left(d s_{\mathbb{R}^{3}}^{2}\right)=\left(1+|g|^{2}\right)^{2}|\omega|^{2} .
$$

Furthermore, $g$ is stereographic projection of the Gauss map of $\Phi_{0}$. This is the Weierstrass representation.

On the other hand, for Weierstrass data $(g, \omega)$ on $D$, we can take $F: D \rightarrow S L(2, \mathbb{C})$ such that

$$
F^{-1} d F=\left(\begin{array}{cc}
g & -g^{2} \\
1 & -g
\end{array}\right) \omega, \quad F\left(z_{0}\right)=\left(\begin{array}{ll}
1 & 0 \\
0 & 1
\end{array}\right)
$$

and set

$$
\Phi_{1}(z):=F(z) \overline{F(z)}^{t} .
$$

Then $\Phi_{1}: D \rightarrow\left\{X \bar{X}^{t} \in \operatorname{Herm}(2) ; X \in S L(2, \mathbb{C})\right\} \cong \mathbb{H}^{3}$ is a CMC 1 immersion with induced metric $\Phi_{1}^{*}\left(d s_{\mathbb{H}^{3}}^{2}\right)=\Phi_{0}^{*}\left(d s_{\mathbb{R}^{3}}^{2}\right)$, where $\mathbb{H}^{3}=\mathbb{H}^{3}(-1)$ is the hyperbolic 3-space with sectional curvature -1 . This is the Bryant representation (Bryant 1987, Umehara and Yamada 1993). $F$ is unique up to the form $A \cdot F, A$ a constant in $S L(2, \mathbb{C})$, so $\Phi_{1}$ is unique up to rigid motions of $\mathbb{H}^{3}$ (see Umehara and Yamada 1993).

This shows that given data $(g, \omega)$ on $D$, we can locally construct a pair of isometric surfaces, a minimal surface $\Phi_{0}(D)$ in $\mathbb{R}^{3}$ and a CMC 1 surface $\Phi_{1}(D)$ in $\mathbb{H}^{3}$ (see Theorem 8 of Lawson 1970).

For both $\Phi_{0}$ and $\Phi_{1}$, the Hopf differential $Q$ on $D$ is defined by $Q=\omega d g$.

DeFINITION 1. Let $M$ be a Riemann surface and $\Phi_{0}: M \rightarrow \mathbb{R}^{3}$ a conformal minimal immersion. Then a CMC 1 immersion $\Phi_{1}: M \rightarrow \mathbb{H}^{3}$ is a cousin surface of $\Phi_{0}$ if

$$
\Phi_{1}^{*}\left(d s_{\mathbb{H}^{3}}^{2}\right)=\Phi_{0}^{*}\left(d s_{\mathbb{R}^{3}}^{2}\right)
$$

holds. We refer to any such pair of surfaces $\Phi_{0}$ and $\Phi_{1}$ as cousins.

The following lemma is immediately obtained from $\S 177$ of Nitsche 1989:

Lemma 2. Let $(g, \omega)$ be the Weierstrass data of a simply-connected CMC 1 surface $\Phi_{1}: D \rightarrow \mathbb{H}^{3}$. Then any cousin minimal surface $\Phi_{0}$ in $\mathbb{R}^{3}$ can be represented (up to a rigid motion) by the Weierstrass data $\left(g, e^{i \theta} \omega\right)$ for some $\theta \in[0, \pi)$.

Recall that a surface has finite topology if it is homeomorphic to a compact Riemann surface $\bar{M}$ with a finite number of points $\left\{p_{1}, \ldots, p_{k}\right\}$ removed, which we write as $M=\bar{M} \backslash\left\{p_{1}, \ldots, p_{k}\right\}$. We have the following proposition, which follows directly from results in Collin et al. 2001 and Sá Earp and Toubiana 2001. 
Proposition 3. Let $M=\bar{M} \backslash\left\{p_{1}, \ldots, p_{k}\right\}$ be a Riemann surface of finite topology, and let $\Phi_{1}: M \rightarrow \mathbb{H}^{3}$ be a conformal CMC 1 immersion with properly embedded annular ends. Let $\Phi_{0}: M \rightarrow \mathbb{R}^{3}$ be a minimal immersion with embedded ends, and assume that $\Phi_{1}$ and $\Phi_{0}$ are cousins. Then the Hopf differentials of $\Phi_{1}$ and $\Phi_{0}$ are holomorphic on $\bar{M}$.

REMARK 4. By Theorem 10 of Collin et al. 2001, all properly embedded annular CMC 1 ends in $\mathbb{H}^{3}$ are conformal to a punctured disk, thus the assumption that $\Phi_{1}$ is conformal is not actually a restriction on the possible choices of $\Phi_{1}$. Because $\Phi_{0}$ and $\Phi_{1}$ are cousins, $\Phi_{0}: M \rightarrow \mathbb{R}^{3}$ is also conformal.

Proof of Proposition 3. Let $\varphi_{1}: \Delta_{\varepsilon}^{*} \rightarrow \mathbb{H}^{3}$ be an arbitrary end of $\Phi_{1}$, where $\Delta_{\varepsilon}^{*}=\{z \in$ $\mathbb{C} ; 0<|z|<\varepsilon\}$ for some $\varepsilon>0$. As noted in Remark 4, we may assume that $\varphi_{1}$ is conformal. Let $\varphi_{0}: \Delta_{\varepsilon}^{*} \rightarrow \mathbb{R}^{3}$ be the corresponding minimal end. By Theorem 10 of Collin et al. 2001, $\varphi_{1}$ has finite total curvature and is regular. Then by Umehara and Yamada 1993, we can take the Weierstrass data associated with $\varphi_{1}$ in the following form:

$$
g(z)=z^{\mu} \hat{g}(z), \quad \hat{g}(0) \neq 0, \quad \omega=z^{v} \hat{w}(z) d z, \quad \hat{w}(0) \neq 0
$$

where $\hat{g}, \hat{w}$ are nonzero holomorphic functions on $\Delta_{\varepsilon}=\{z \in \mathbb{C} ;|z|<\varepsilon\}$, and $\mu, v \in \mathbb{R}, \mu>0$, $v \leq-1, \mu+v \in \mathbb{Z}, \mu+v \geq-1$.

By Lemma 2, there exists a $\theta \in[0, \pi)$ such that $\left(g, e^{i \theta} \omega\right)$ is the Weierstrass data associated with $\varphi_{0}$. Because $g$ is stereographic projection of the Gauss map of $\varphi_{0}, g$ is well-defined on $\Delta_{\varepsilon}^{*}$, so $\mu \in \mathbb{N}$ and hence $-v \in \mathbb{N}$.

The first and second coordinates of $\varphi_{0}$ are

$$
\operatorname{Re} \int_{z_{0}}^{z}\left(1-g^{2}\right) e^{i \theta} \omega, \quad-\operatorname{Im} \int_{z_{0}}^{z}\left(1+g^{2}\right) e^{i \theta} \omega,
$$

and $\varphi_{0}$ is asymptotic to a catenoid or planar end, by Schoen 1983. Also $g(0)=0$, and the limiting normal of the end $\varphi_{0}$ must be vertical. Therefore, $v$ must be -2 for the end to be embedded, and $\hat{w}^{\prime}(0)$ must be 0 for the end $\varphi_{0}$ to be well-defined on $\Delta_{\varepsilon}^{*}$.

Lemma 2.4 of Sá Earp and Toubiana 2001 showed that $0 \neq \hat{g}(0) \hat{w}(0)=\left(1-\mu^{2}\right) / 4 \mu$. So $\mu$ cannot be 1 because $\hat{g}(0) \neq 0$ and $\hat{w}(0) \neq 0$. Furthermore, Lemma 2.9 of Sá Earp and Toubiana 2001 showed that

$$
\hat{w}^{\prime}(0)= \begin{cases}2 \hat{w}(0)^{2} \hat{g}(0) & \text { if } \mu=2, \\ 0 & \text { if } \mu \geq 3 .\end{cases}
$$

So $\mu$ cannot be 2 . Therefore $\mu \geq 3$.

Thus the Hopf differentials $\omega d g$ and $e^{i \theta} \omega d g$ have order $\mu+v-1 \geq 0$ at $z=0$. Hence they are holomorphic at each end, as well as on $M$ itself.

An end $\varphi_{0}: \Delta_{\varepsilon}^{*} \rightarrow \mathbb{R}^{3}$ (resp. $\varphi_{1}: \Delta_{\varepsilon}^{*} \rightarrow \mathbb{H}^{3}$ ) is said to be a planar end (resp. horosphere end) if $\mu+v \geq 0$. So we have the following corollary: 
Corollary 5. Hypotheses being as in Proposition 3, then $\Phi_{0}$ has only planar ends and $\Phi_{1}$ has only horosphere ends.

Corollary 6. Let $M=\bar{M} \backslash\left\{p_{1}, \ldots, p_{k}\right\}$ be a Riemann surface of finite topology so that $\bar{M}$ has genus zero. Let $\Phi_{0}: M \rightarrow \mathbb{R}^{3}, \Phi_{1}: M \rightarrow \mathbb{H}^{3}$ be properly immersed cousin surfaces with embedded ends. Then $\Phi_{0}$ is a plane and $\Phi_{1}$ is a horosphere.

Proof. Since there exists no nonzero holomorphic 2-differential on the sphere $\mathbb{C} \cup\{\infty\}$, the Hopf differential is identically zero. So both $\Phi_{0}(M)$ and $\Phi_{1}(M)$ are totally umbilic. Therefore $\Phi_{0}$ is a plane and $\Phi_{1}$ is a horosphere.

Corollary 7. Let $M=\bar{M} \backslash\left\{p_{1}, \ldots, p_{k}\right\}$ be a Riemann surface of finite topology so that $\bar{M}$ has genus $\gamma$. Let $\Phi_{0}: M \rightarrow \mathbb{R}^{3}, \Phi_{1}: M \rightarrow \mathbb{H}^{3}$ be properly immersed cousin surfaces with embedded ends, and suppose they have total curvature more than $-16 \pi$. Then $\Phi_{0}$ is a plane and $\Phi_{1}$ is a horosphere.

Proof. Lopez 1992 showed that any minimal surface with total curvature $-4 \pi$ or $-8 \pi$ has a non-holomorphic Hopf differential $Q$ on $\bar{M}$. Thus the only possibility (other than a plane) is that $\Phi_{0}: M \rightarrow \mathbb{R}^{3}$ is a properly immersed minimal surface with embedded planar ends and total curvature $-12 \pi$. By Theorem 4 of Jorge and Meeks 1983, each end of $\Phi_{0}$ is embedded if and only if

$$
\int_{M} K d A=-4 \pi(k+\gamma-1)
$$

holds, where $K$ and $d A$ are the Gaussian curvature and the area element of $\Phi_{0}$. So $k+\gamma=4$. Since any complete minimal surface with finite total curvature and one embedded end is a plane, and since the only complete minimal surface in $\mathbb{R}^{3}$ with finite total curvature and two embedded ends is the catenoid (Schoen 1983), $\Phi_{0}(M)$ is a torus with three embedded planar ends. But Theorem 26 of Kusner and Schmitt 1992 showed that such a surface does not exist, completing the proof.

Corollary 8. Let $M=\bar{M} \backslash\left\{p_{1}, \ldots, p_{k}\right\}$ be a Riemann surface of finite topology so that $\bar{M}$ has genus one. Let $\Phi_{0}: M \rightarrow \mathbb{R}^{3}, \Phi_{1}: M \rightarrow \mathbb{H}^{3}$ be properly immersed cousin surfaces with embedded ends. Then $\Phi_{0}$ and $\Phi_{1}$ each have at least 4 ends.

Proof. By Theorem 4 of Jorge and Meeks 1983 again, the right hand side of (1) is $-4 k \pi$. So $k \geq 4$, by Corollary 7 .

REMARK 9. Theorem 3 of Miyaoka and Sato 1994 found examples of complete minimal surfaces of genus one with four embedded ends, but they all contain non-planar ends.

Remark 10. Costa 1993 and Kusner and Schmitt 1992 found examples of complete minimal surfaces of genus one with four embedded planar ends. But none of them satisfies the condition that the Hopf differential extends holomorphically to the ends. 
Defining annular ends to be those which are homeomorphic to punctured disks, Theorem 12 of Collin et al. 2001 showed that each end of a properly embedded non-totally-umbilic CMC 1 surface $\Phi_{1}: M \rightarrow \mathbb{H}^{3}$ with annular ends is asymptotic to an end of a CMC 1 catenoid. In particular, such a surface does not have horosphere ends. We saw in the proof of Proposition 3, in conjunction with Remark 4, that any single embedded annular end asymptotic to a CMC 1 catenoid in $\mathbb{H}^{3}$ cannot have a corresponding minimal cousin in $\mathbb{R}^{3}$ with an embedded end. Hence, $\Phi_{1}$ does not have a cousin $\Phi_{0}: M \rightarrow \mathbb{R}^{3}$ with embedded ends. So we have the following corollary, in which we do not need to assume that $M$ has finite topology, since finite topology was not assumed in Theorem 12 of Collin et al. 2001:

COROLlaRY 11. Let $M$ be a Riemann surface. Let $\Phi_{1}: M \rightarrow \mathbb{H}^{3}$ be a conformal CMC 1 proper embedding with annular ends, and let $\Phi_{0}: M \rightarrow \mathbb{R}^{3}$ be a minimal surface with embedded ends. Assume that $\Phi_{1}$ and $\Phi_{0}$ are cousins. Then $\Phi_{0}$ is a plane and $\Phi_{1}$ is a horosphere.

REMARK 12. Regarding Corollary 11:

(i) If the assumption that $\Phi_{1}$ is embedded is removed, then the pair of cousin surfaces given by the Weierstrass data

$$
(g, \omega)=\left(z, \frac{n^{2}-1}{4} z^{-2} d z\right) \quad \text { on } \quad M=\mathbb{C} \backslash\{0\}, \quad n \in \mathbb{N} \backslash\{1\}
$$

is a counterexample. In fact, each end of $\Phi_{1}$ in this example is an $n$-fold cover of an embedded end, and $\Phi_{0}$ is an embedding.
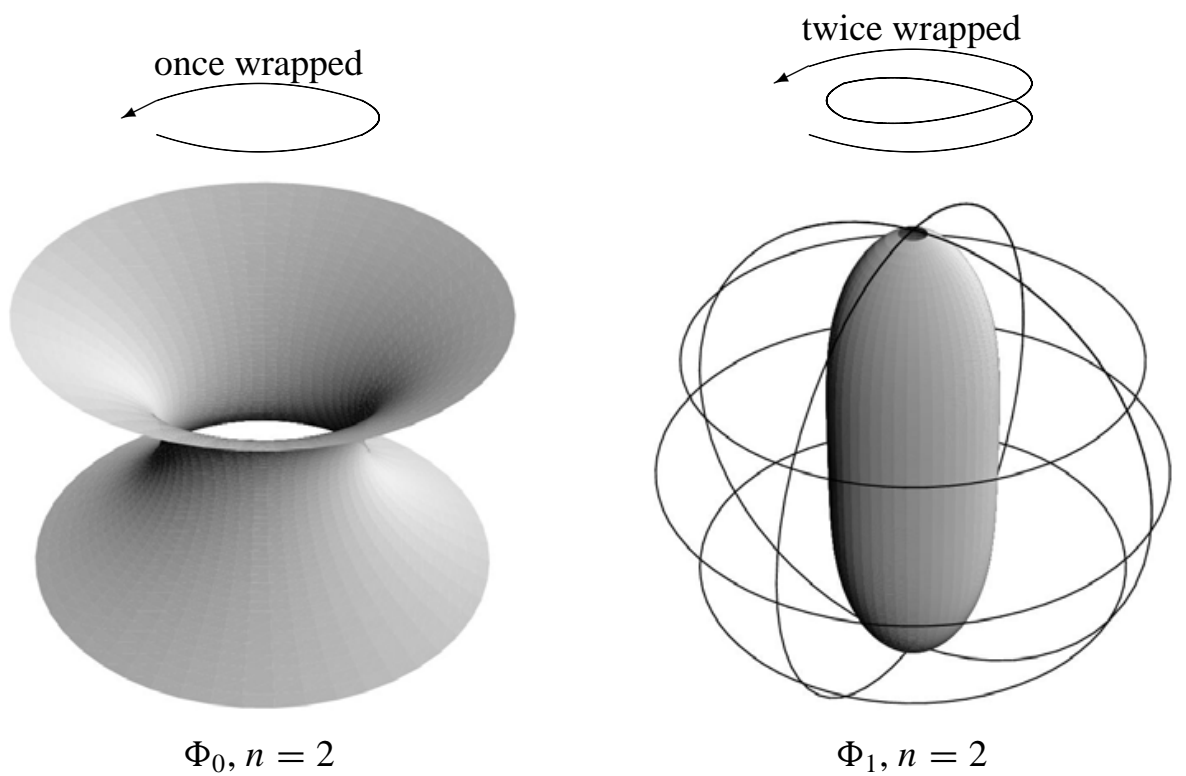

Fig. 1 - The surfaces $\Phi_{0}$ and $\Phi_{1}$ in (i) of Remark 12 . 
(ii) If the assumption that $\Phi_{1}$ is embedded is replaced with the weaker assumption that only the ends are embedded, then any possible counterexamples can not satisfy the conditions of Corollaries 6 or 7 or 8 .

(iii) If the weaker assumption in (ii) is used, and the assumption that the ends of $\Phi_{0}$ are embedded is removed, then the pair of cousin surfaces given by the Weierstrass data

$$
(g, \omega)=\left(z^{n}, \frac{1-n^{2}}{4 n} z^{-1-n} d z\right) \quad \text { on } \quad M=\mathbb{C} \backslash\{0\}, \quad n \in \mathbb{N} \backslash\{1\}
$$

is a counterexample to the corollary. In fact, in this example, each end of $\Phi_{1}$ is embedded, and each end of $\Phi_{0}$ is an $n$-fold cover of an embedded end.

(iv) If the assumption that $\Phi_{1}$ is embedded is kept, but the assumption that each end of $\Phi_{0}$ is embedded is removed, then the author does not know of any counterexamples to the corollary.
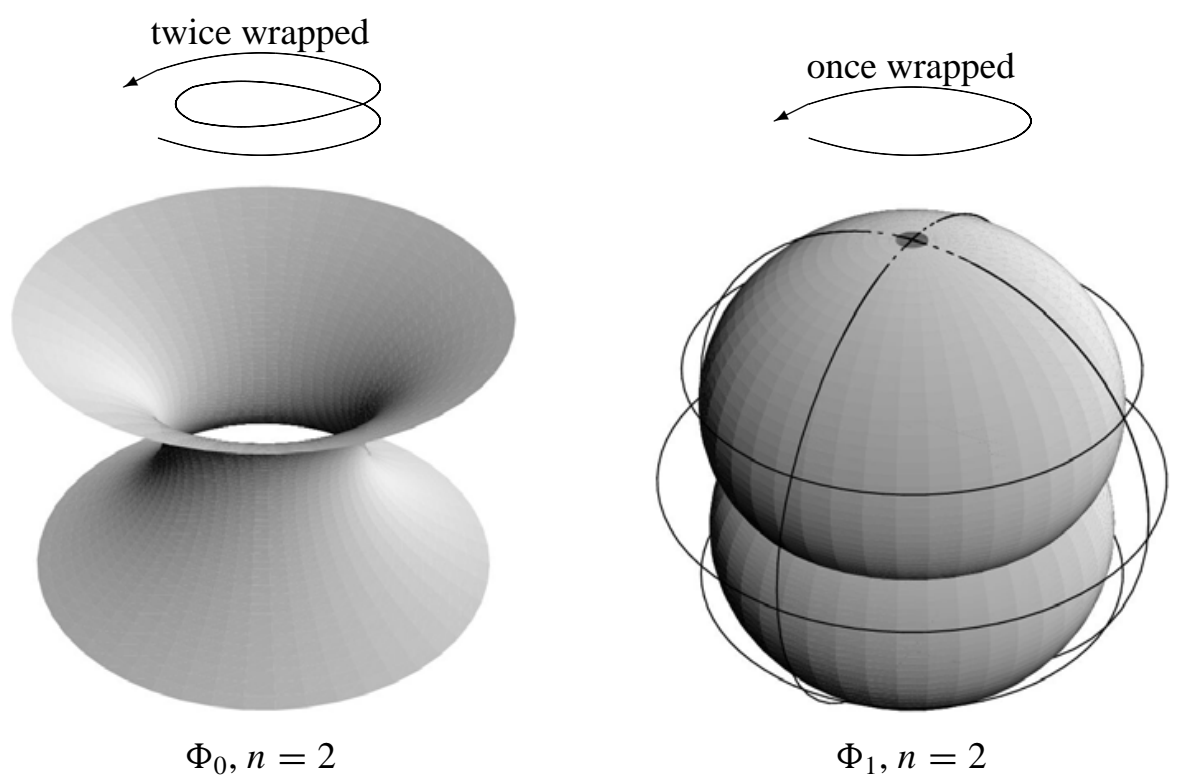

Fig. 2 - The surfaces $\Phi_{0}$ and $\Phi_{1}$ in (iii) of Remark 12.

REMARK 13. Theorem 3.3 of Choi et al. 1990 showed that a properly embedded minimal surface in $\mathbb{R}^{3}$ which has more than one end is minimally rigid. Corollary 3.4 of Umehara and Yamada 1992 showed that if cousin surfaces $\tilde{\Phi}_{c}: \Delta_{\varepsilon} \rightarrow \mathbb{H}^{3}\left(-c^{2}\right)(c>0)$ associated with a minimal surface $\Phi_{0} \circ \rho: \Delta_{\varepsilon} \rightarrow \mathbb{R}^{3}$ are well-defined on $\Delta_{\varepsilon}^{*}$ for all $c$, then all of the surfaces in the associate family of $\Phi_{0}$ are well-defined on $\Delta_{\varepsilon}^{*}$, where

$$
\rho: \Delta_{\varepsilon} \ni z \mapsto \varepsilon e^{(z-\varepsilon) /(z+\varepsilon)} \in \Delta_{\varepsilon}^{*}
$$


is the projection. However, this cannot lead us to another proof of Corollary 11, because we only assume that the $\Phi_{c}$ is well-defined when $c=0,1$. Furthermore, we allow $M$ to have positive genus, so we are not considering well-definedness merely on domains which are simply-connected or homeomorphic to $\Delta_{\varepsilon}^{*}$.

\section{ACKNOWLEDGMENTS}

The author thanks Professors Wayne Rossman and Hitoshi Furuhata for their assistance with this work.

\section{RESUMO}

Mostramos que as diferenciais de Hopf de um par de superfícies primas, a saber, uma superfície mínima em um espaço euclideano de dimensão 3 e uma superfície de curvatura média constante (CMC) um em um espaço hiperbólico de dimensão 3, se estendem holomorficamente em cada fim. Usando este resultado, obtemos condições para que o par seja um plano e uma horosfera.

Palavras-chave: superfícies mínimas, prismas de CMC 1, espaços hiperbólicos.

\section{REFERENCES}

BRYANT R. 1987. Surfaces of Mean Curvature One in Hyperbolic Space, Astérisque 154-155: 321-347.

Choi HI, Meeks III WH And White B. 1990. A rigidity theorem for properly embedded minimal surfaces in $\mathbb{R}^{3}$, J Diff Geom 32: 65-76.

Collin P, Hauswirth L and Rosenberg H. 2001. The geometry of finite topology Bryant surfaces, Ann of Math 153: 623-659.

Costa CJ. 1993. Complete minimal surfaces in $\mathbb{R}^{3}$ of genus one and four planar embedded ends, Proc of Amer Math Soc 119: 1279-1287.

Jorge L And Meeks III WH. 1983. The topology of complete minimal surfaces of finite total Gaussian curvature, Topology, 22: 203-221.

KUSNER R AND SCHMitT N. 1992. The spinor representation of minimal surfaces, G.A.N.G. preprint [3.27].

Lawson HB. 1970. Complete minimal surfaces in $S^{3}$, Ann of Math 92: 335-374.

LOPEZ FJ. 1992. The classification of complete minimal surfaces with total curvature greater than $-12 \pi$, Trans Amer Math Soc 334: 49-74.

MiyaOKa R and Sato K. 1994. On complete minimal surfaces whose Gauss maps miss two directions, Arch Math 63: 565-576.

Nitsche JCC. 1989. Lectures on minimal surfaces, Vol.1, Cambridge University Press.

Rossman W, Umehara M and Yamada K. 1997. Irreducible constant mean curvature 1 surfaces in hyperbolic space with positive genus, Tohoku Math J 49: 449-484. 
Rossman W, Umehara M and Yamada K. 2001. Period problems for mean curvature one surfaces in $\mathbb{H}^{3}$ (with application to surfaces of low total curvature), to appear in NSJ-IRI Tokyo proceedings.

SÁ EARP R AND Toubiana É. 2001. On the geometry of constant mean curvature one surfaces in hyperbolic space, Illinois J Math 45: 371-401.

SCHOEN R. 1983. Uniqueness, symmetry and embeddedness of minimal surfaces, J Diff Geom 18: 791-809.

Umehara M and Yamada K. 1992. A parametrization of the Weierstrass formulae and perturbation of complete minimal surfaces in $\mathbb{R}^{3}$ into the hyperbolic 3-space, J Reine Angew Math 432: 93-116.

Umehara M and Yamada K. 1993. Complete surfaces of constant mean curvature 1 in the hyperbolic 3-space, Ann of Math 137: 611-638.

Yu Z. 2001. Surfaces of constant mean curvature one in the hyperbolic three-space with irregular ends, Tohoku Math J 53: 305-318. 